\title{
Epidemiology of tuberculosis in Eastern Sudan
}

\author{
Tajeldin M Abdallah, Abdel Aziem A Ali* \\ Faculty of Medicine, Kassala University, Sudan
}

\section{PEER REVIEW}

Peer reviewer

Prof. Abdalla Ali Mohammed, Department of Obstetrics \& Gynecology, Postgraduate Dean.

Tel: +249912233781

E-mail:dohmaa@yahoo.co.uk

Comments

This is a well designed study, addressing an important health problem in the local community. Authors are aware of the limitation of their study. If this is considered, a prospective study well answer many queries arised from this study.

(Details on Page 1000)

\begin{abstract}
Objective: To investigate the epidemiological factors of tuberculosis (TB) in eastern Sudan. Methods: The socio-demographic and clinical data was retrieved from the database at Kassala hospital during the year of 2011. The medical file of consequent patients who was discharged from the same ward in the hospital was reviewed to act as control for the TB patients. Results: A total of 670 patients were registered at Kassala hospital with clinical, laboratory and radiological evidence proven TB. Pulmonary TB accounted for $73.4 \%$ while extra-pulmonary TB was reported in 26.6\% of all TB patients. The mean age (SD) was not significantly different between the cases and controls (670 in each arm). TB patients were those who had less education, and the infection more likely common among male patients. Conclusions: Intervention from outside the health field in particular awareness of associated risk factors and improvement of the educational level potentially will strengthen TB control.
\end{abstract}

\section{KEYWORDS}

Tuberculosis, Epidemiology, Mycobacterium, Sudan

\section{Introduction}

Tuberculosis (TB) remains a major health problem in low income countries[1]. During 2008 there were 8.9-9.9 million reported TB cases all over the world, most of them in Africa and Asia[2]. Control of TB is a high priority and becomes one of the international missions after the increase in the number of cases all over the world including the developed countries[3]. TB was declared as a global public health emergency since 1993 by the World Health Organization[4]. Identification of epidemiological and risk factors is fundamental and crucial for policy makers to put the appropriate strategies to prevent the disease and decrease the global burden of TB. Eastern Sudan is an endemic area of TB[5] and to our knowledge no previous study has documented the epidemiology of the disease, thus the current study directed to investigate the epidemiology and associated risk factors among TB patients.

\section{Materials and methods}

To investigate the epidemiological factors of TB, the socio-demographic and clinical data was retrieved from the database at Kassala hospital during 1st January and 31st December 2011. This database is based on voluntary registration at the chest department of Kassala hospital, eastern Sudan which is an endemic area of TB. The data contains the personal and social information, the clinical presentation, type of $\mathrm{TB}$, follow up and the outcome. The outcome registered as complete recovery, relapse, lost during follow up and death and the death is registered without further explanation (TB related or not TB related).

\footnotetext{
*Corresponding author: Abdel Aziem A Ali, P.O. Box 496, Department of Obstetrics and Gynecology, Faculty of Medicine, Kassala University, Kassala, Sudan.

Tel: +249912163820

Fax: +249411823501

E-mail: abuzianab73@yahoo.com
}

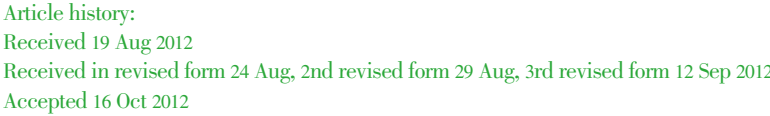


For all identified TB patients the WHO guidelines are applied, this guidelines is based on the internationally agreed strategies for TB control (The Directly Observed Treatment Short Course or DOTS)[6]. The regime which is recommended at this hospital consists of Isoniazid, Rifampicin, Pyrazinamide and Ethambutol for the intitial phase then Isoniazid, Rifampicin and Pyrazinamide for the continuation phase.

The medical record of consequent patients who was treated and discharged from the same department in the hospital was reviewed to act as control for the TB cases.

Data were entered into a computer database and SPSS software (SPSS Inc., Chicago, USA, version 13.0) and double checked before analysis. Means and proportions for the socio-demographic characteristics were compared between the groups of the study (cases and controls) and $P<0.05$ was considered significant.

The study received ethical clearance from the Research Board at Ministry of Health Kassala State, Eastern Sudan.

\section{Results}

During the year of 2011, a total of 670 patients were registered at Kassala hospital with clinical, laboratory and radiological evidence proven TB. Their mean age (SD) was 34.1 (18) years. The majority of these patients had less than secondary education, male and of rural residence. Of the total patients, $36.8 \%$ were non skill workers, $26.1 \%$ skill workers, $25.4 \%$ employees, and $11.7 \%$ were house wives. Pulmonary TB accounted for $73.4 \%$ (492/670) while extra-pulmonary TB was reported in $26.6 \%$ (178/670) of all TB patients. The mean age (SD) was not significantly different between the cases and controls (670 in each arm), 34.1 (18.0) vs. 33.8 (8.5), $P=0.7$. TB patients were those who had less education, and the infection more likely common among male patients (Table 1).

Table 1

Epidemiological factors of TB patients, Eastern Sudan 2008-2010 $(n=670)$.

\begin{tabular}{lccc}
\hline Variables & Cases & Control & $P$ \\
Sex male & $427(63.7 \%)$ & $354(52.8 \%)$ & 0.0 \\
Rural residence & $433(64.6 \%)$ & $450(67.2 \%)$ & 0.2 \\
Education s secondary & $445(66.4 \%)$ & $351(52.4 \%)$ & 0.0 \\
Occupation non skill worker & $245(36.8 \%)$ & $254(38.1 \%)$ & 0.7 \\
\hline
\end{tabular}

Five hundred fifty $(82.1 \%), 91(13.6 \%), 19(2.8 \%)$ and 10 $(1.5 \%)$ of these $670 \mathrm{~TB}$ patients completely recovered, lost during follow up, died and relapsed, respectively.

\section{Discussion}

Worldwide there is increased focus on addressing epidemiological and risk factors of TB[7,8]. Intervention from outside the health field in particular awareness of associated risk factors and social protection potentially will strengthen TB control. In the current study, illiteracy and male patients were the predictors for TB. During the year 2010 more cases of TB were reported than ever before and interestingly these cases continue to cluster among those who were poor, Hungary and of ethnic minorities[9,10]. Over the last few decades the epidemiological and risk factors for TB have changed dramatically, poverty and malnutrition strongly associated with the infection[11]. Smoking, which is unusual habits among female in Sudan, was reported to enhance the risk of TB infection and this might explained its high risk among male patients[12-18]. Furthermore, research on TB underlying cellular mechanism suggested that cathelicidin, an antimicrobial peptide regulated by Vitamin D receptor have been linked to the disease susceptibility[19]. This fact again might explain the gender difference as a risk factor for the disease in our study area, since females are less likely to expose to sunlight in comparison to males. Traditionally men have had higher incidence rate of TB than women, however in contrast to our study recent report carried out among all TB black patients, women had highest proportion of extra-TB[20]. There is strong relation between TB and HIV notably in sub-Saharan Africa, globally one in seven TB cases is associated with HIV infection[11], thus the social and associated risk factors of HIV infection also act as indirect determinants for TB[21].

Our study had several limitations; it was a hospitalbased one; which might not reflect what was at the community level. The HIV status was not yet tested and the diagnosis of extra-pulmonary TB in most time based on clinical interpretation however all of the cases in our study were confirmed TB patients. Moreover the patients' database didn't include any information on nutritional status, smoking and alcohol consumption.

\section{Conflict of interest statement}

We declare that we have no conflict of interest.

\section{Acknowledgements}

We are grateful to the staff of Kassala Teaching Hospital for their assistance in data retrieval.

\section{Comments}

\section{Background}

Tuberculosis continues to be an important public health problem worldwide and Sudan characterized by high prevalence rate of tuberculosis. In Eastern Sudan, Ali et al. reported an incidence rate of 275/100000 during pregnancy. Moreover extrapulmonary tuberculosis is a very common health problem in Eastern Sudan and there is a high incidence rate of female genital tuberculosis $(0.9 \%)$ With chronic pelvic pain as the main clinical presentation. 
Most epidemiological research on tuberculosis, nowadays concentrate on its relation to HIV/AIDS. Away from this, epidemiology of pulmonary and extra-pulmonary tuberculosis in this highly endemic area needs further study.

\section{Research frontiers}

This study utilizes a routinely collected data and draw important epidemiological findings about TB. This type of study is much important as it highlights the area for adjusting local or national control measures. A wider community based epidemiological study addressing the above concerns is be the recommendation of this paper.

\section{Related reports}

Authors related high incidence of TB among male to smoking. (WHO Global Tuberculosis Report 2012). Tuberculosis is more common in men than in females with a wide variation between countries.

\section{Innovations and breakthroughs}

In developing countries, epidemiological surveys is expensive and faced with many constraints. Extracting epidemiological data from hospital based records is a less expensive source, which is well utilized in this study. This paper is first epidemiological study in Kassala State.

\section{Applications}

Epidemiological studies are more important for local policy makers regarding tuberculosis control. Vulnerables groups are highlighted. This study is highly applicable in this respect.

\section{Peer review}

This is a well designed study, addressing an important health problem in the local community. Authors are aware of the limitation of their study. If this is considered, a prospective study well answer many queries arised from this study.

\section{References}

[1] Maartens G, Wilkinson RJ. Tuberculosis. Lancet 2007; 370: 2030-2043.

[2] World Health Organization. Global tuberculosis control: a short update to the 2009 report. 3rd WHO annual report on global TB control; 1999.

[3] Alfaresi MS, Hag-Ali M. Susceptibility pattern and epidemiology of Mycobacterium tuberculosis in United Emirati Hospital. Open Microbiol J 2010; 4: 1-4.

[4] Alfaresi MS, Abdulsalam AI, Elkoush A. Comparison of the automated Cobas amplicor mycobacterium assay with the conventional methods for direct detection of Mycobacterium tuberculosis complex in respiratory and extrapulmonary specimens. Saudi Med J 2006; 27(9): 1346-1351.

[5] Abdallah TM, Ali AA, Adam I. Provider-intiated HIV testing and counselling among tuberculosis patients in Kassala, eastern Sudan. J Infect Public Health 2012; 5(1): 63-66.
[6] World Health Organization. The stop TB strategy. Geneva: World Health Organization; 2006. [Online] Available from http:// www.stoptb.org/assets/documents/resources/publications/plan_ strategy/ The Stop TB Strategy. Final pdf. [Accessed on Nov. 2011].

[7] Hargreaves JR, Boccia D, Evans CA, Adato M, Petticrew M, Porter JDH. The social determinants of tuberculosis; from evidence to action. Am J Public Health 2011; 101(4): 654-662.

[8] Osman E, Daniel O. Prevalence and risk factors associated with drug resistant TB in South West, Nigeria. Asian Pac J Trop Med 2011; 4(2): 148-151.

[9] Pakasi TA, Karyadi E, Dolmans WM, van der Meer JW, van der Velden K. Malnutrition and sociodemographic factors associated with pulmonary tuberculosis in Timor and Rote Islands, Indonesia. Int J Tuberc Lung Dis. 2009; 13(6): 755-759.

[10] Stout JE, Saharia KK, Nageswaran S, Ahmed A, Hamilton CD. Racial and ethnic disparities in pediatric tuberculosis in North Carolina. Arch Pediatr Adolesc Med 2006; 160(6): 631-637.

[11] Thaiss WM, Thaiss CC, Thaiss CA. Recent development in the epidemiology and management of tuberculosis-new solutions to old problem. Infect Drug Resist 2012; 5: 1-8.

[12] Bates MN, Khalakdina A, Pia M, Chang L, Lessa F, Smith KR. Risk of tuberculosis from exposure to tobacco smoke: a systemic review and meta-analysis. Arch Int Med 2007; 167(4): $335-342$.

[13] Singh S, Jain P, Aggarwal G, Dhiman P, Singh S, Sen R. Primary hepatic tuberculosis: A rare but fatal clinical entity if undiagnosed. Asian Pac J Trop Med 2012; 5(6): 498-499.

[14] Singal R, Gupta S, Gupta S. Primary abdominal tuberculosis presenting as peritonitis in a young child-managed surgically. Asian Pac J Trop Med 2012; 5(5): 413-415.

[15] Johnkennedy N, Onyinyechi AS, Chukwunyere NE. The antioxidant status and lipid peroxidation product of newly diagnosed and 6 weeks follow-up patients with pulmonary tuberculosis in Owerri, Imo state, Nigeria. Asian Pac J Trop Med 2012; 4(11): 292-294.

[16] Dhamgaye TM, Mishra G, Deokar K. Miliary tuberculosis with bilateral pneumothorax - A case report. Asian Pac J Trop Dis 2012; 2(6): 492-494.

[17] Kataria SP, Singh S, Tanwar P, Kumar S, Singh G. Primary tuberculosis of the tongue in an immunocompetent patient: A case report. Asian Pac J Trop Dis 2012; 2(1): 78-79.

[18] Suraiya S, Musa M, Suppian R, Haq JA. Serological diagnosis for active tuberculosis in Malaysian population: Comparison of four protein candidate. Asian Pac J Trop Dis 2012; 2(Suppl 1): S312-S315.

[19] Liu PT, Stenger S, tang DH, Modlin RL. Cutting edge: vitamin D-mediated human antimicrobial activity against Mycobacterium tuberculosis is dependent on the induction of cathelicidin. J Immunol 2007; 179(4): 2060-2063.

[20] Martinez AN, Rhee JT, Small PM, Behr MA. Sex differences in the epidemiology of tuberculosis in San Francisco. Int J Tuberc Lung Dis 2000; 4: 26-31.

[21] Khosravi AD, Alavi SM, Hashemzade M, Abasi E, Seghatoleslami S. The relative frequency of Mycobacterium tuberculosis and Mycobacterium avium infections in HIV positive patients, Ahvaz, Iran. Asian Pac J Trop Med 2012; 5(1): 71-74. 